this hypothesis, but the lack of a similar correlation for IgG and IgA suggests that other factors may be important, one possibility being a general increase in immune responsiveness.

The prevalence of autoantibodies in the present series belies the generally accepted view that they are rarely, if ever, found in alcoholic cirrhosis. ${ }^{16}$ They were less prevalent in our patients than in those with chronic active hepatitis ${ }^{17}$ but more prevalent than in the controls. Furthermore, although the titres were generally low, similar titres are found in some patients with chronic active hepatitis. ${ }^{17}$

We have suggested that the higher prevalence of HLA-B8 in chronic active hepatitis occurs because this antigen is linked to genes that promote abnormally raised and prolonged antibody responses. ${ }^{10}$ While HLA-B8 is also fairly prevalent $(45 \%)$ in alcoholic cirrhosis, the association is not as great as in HBsAgnegative chronic active hepatitis, in which more than $60 \%$ of the patients have this histocompatibility antigen. ${ }^{18}$ Similarly, the higher prevalence of serum autoantibodies and raised immunoglobulin concentrations is not as striking as in chronic active hepatitis, ${ }^{17}$ and thus immunologically these two groups of patients seem to differ in the intensity of their immune responses. The biological significance of the absence of HLA-A28 is not clear, but this antigen was also not found in any of our patients with $\mathrm{HBsAg}$-negative chronic active hepatitis ${ }^{8}$ nor in those originally reported by MacKay and Morris. ${ }^{9}$

In spite of these genetic similarities there are obvious clinical and histological differences between the two diseases, and there are probably qualitative, as well as quantitative, differences in their immunopathogenesis. Thus, $T$ lymphocytes were recently found to predominate $(91 \%)$ in the portal tract infiltrates in alcoholic liver disease, whereas the proportion in patients with chronic active hepatitis seemed to be much lower. ${ }^{18}$ Possibly these $T$ lymphocytes are reacting to the changes that alcohol may induce in hepatocyte membranes ${ }^{19}$ and are actively implicated in the destruction of these changed liver cells. The intensity of this reaction may determine the rate of progression to cirrhosis, and any factor that may be associated with unusually intense immune responses, such as high immunoglobulin concentrations, autoantibodies, and possibly HLA-B8, would therefore be expected to be particularly common in those who have developed cirrhosis.

We are indebted to the Wellcome Trust for their continued generous support and to Dr Bernard Portmann for the histological assessments. RJB was supported by the Canadian Hepatic Foundation.

\section{References}

1 Rubin, E, and Lieber, C S, Clinics in Gastroenterology, 1975, 4, 247.

2 Maddrey, W C, and Iber, F L, Annals of Internal Medicine, 1964, 61, 667.

3 Galambos, J T, Gastroenterology, 1972, 63, 1026.

4 Mikes, A A, et al, Lancet, 1975, 1, 951.

5 Cochrane, A M G, et al, Gut, 1975, 16, 400.

${ }^{6}$ Miller, J G, et al, Lancet, 1972, 2, 296.

7 Thomson, A D, et al, Nature New Biology, 1974, 252, 271.

${ }^{8}$ Galbraith, R M, et al, British Medical fournal, 1974, 3, 604.

${ }^{9}$ MacKay, I R, and Morris, P J, Lancet, 1972, 2, 793.

10 Galbraith, R M, et al, Lancet, 1976, 1, 930.

11 Batchelor, J R, in Handbook of Experimental Immunology, ed D M Weir, p 32. Oxford, Blackwell, 1973.

12 Roitt, T M, and Doniach, D, Manual of Autoimmune Serology, Geneva, World Health Organisation, 1969.

${ }^{13}$ Mancini, G, Carbonara, A D, and Heremans, J F, Immunochemistry, 1965, $2,235$.

${ }^{14}$ Eddleston, A L W F, et al, Gut, 1969, 10, 711.

15 Triger, D R, et al, Lancet, 1973, 1, 1494.

${ }^{16}$ Sherlock, S, Clinics in Gastroenterology, 1975, 4, 281.

17 Doniach, D, and Walker, J G, in Progress in Liver Disease, ed H Popper and F Schaffner, p 385. New York, Grune and Stratton, 1972.

${ }^{18}$ Husby, G, et al, fournal of Clinical Investigation, 1975, 56, 1198.

19 Peters, W, et al, Gut, 1975, 16, 826.

\title{
Effect of ethyloestrenol on fibrinolysis in the vessel wall
}

\author{
U HEDNER, I M NILSSON, S ISACSON
}

The spontaneous fibrinolytic activity, local fibrinolytic activity during standardised venous occlusion of the arms, and fibrinolytic activity of the vessel walls increased significantly after treatment with ethyloestrenol $8 \mathrm{mg} /$ day for three months. No further increase occurred after three months, and ethyloestrenol $4 \mathrm{mg} /$ day had no effect. No values rose significantly in the patients with a normal fibrinolytic system. One patient suffered a recurrence within three months of treatment, before the fibrinolytic system became normal. In one patient the fibrinolytic defect reappeared after 10 months in spite of continued treatment. Two of the three women of fertile age developed irregular cycles and intermenstrual bleeding, which disappeared when the treatment was withdrawn. No other side effects were observed.

\section{Introduction}

A defective fibrinolytic defence mechanism (decreased fibrinolytic activator activity in the vein walls or a defective release of fibrinolytic activator from the vein walls, or both) has been found in about $70 \%$ of patients with recurrent deep venous thrombosis (DVT) and no known predisposing condition. ${ }^{1}$ Fearnley $e t \mathrm{al}^{2}$ showed that phenformin $(100 \mathrm{mg} /$ day $)$ combined with ethyloestrenol $(8 \mathrm{mg} /$ day) increased the spontaneous fibrinolytic activity of the blood. The same treatment was later

Coagulation Laboratory, Allmänna Sjukhuset, Malmö, and Department of Surgery, Halmstad, Sweden

U HEDNER, MD, associate professor, coagulation laboratory

I M NILSSON, MD, professor of medicine, coagulation laboratory

$S$ ISACSON, MD, associate professor, department of surgery 
shown to increase the fibrinolytic activity in the vein walls when given to healthy volunteers. ${ }^{3}$ Furthermore, when given to patients with recurrent DVT and a defective fibrinolytic defence mechanism treatment with phenformin and ethyloestrenol increases the spontaneous fibrinolytic activity, the capacity to release fibrinolytic activator during venous occlusion of the arms, and the fibrinolytic activity of the vessel walls. ${ }^{4}$ Anabolic steroids alone have also been shown to increase the venous plasma fibrinolytic reserve..$^{56}$

The side effects of phenformin are, however, serious, so the effect on the fibrinolytic system of ethyloestrenol alone in doses of $8 \mathrm{mg} /$ day or $4 \mathrm{mg}$ /day was studied in 49 patients with recurrent DVT.

\section{Patients and methods}

The 49 patients ( 34 men, 15 women) had either decreased fibrinolytic activity in the vessel walls or a decreased release mechanism, or both. Their ages ranged from 22 to 78 years (mean 51.3), and 10 were aged under 40 . None of the patients had any coexisting disease. Forty-five of the patients had had phlebographically verified recurrent DVT during the five years before the first examination (two to five thrombotic episodes), and five also had had pulmonary embolism. The remaining four patients had arterial thrombosis (thrombi were present in the internal carotid artery or middle cerebral artery or the patients suffered transient ischaemic attacks or peripheral arterial ischaemia). On admission to the trial 10 of the patients were receiving dicoumarol prophylactically, and this was continued until the fibrinolytic system returned to normal. Six men had been treated with the combination of phenformin and ethyloestrenol ${ }^{4}$ for one to four years.

The patients were examined on average three months after the end of their last thrombotic episode. Each patient was examined on two consecutive days and 21 were re-examined just before the beginning of treatment to check that the defect was consistent. All patients were then examined after three, six, and 10 to 17 months of treatment (table I).

TABLE I-Number of patients treated and followed up

\begin{tabular}{l|c|c|c}
\multicolumn{1}{r|}{ Dose : } & $8 \mathrm{mg} /$ day & $4 \mathrm{mg} /$ day & $8 \mathrm{mg} /$ day* \\
\hline No of patients & 31 & 12 & 6 \\
No of patients examined at: & 29 & 10 & 3 \\
3 months & 15 & 4 & 5 \\
10-17 months & &
\end{tabular}

*These patients had earlier received phenformin and ethyloestrenol together.

Thirty-one of the patients were given ethyloestrenol $8 \mathrm{mg} /$ day, and 12 received $4 \mathrm{mg} /$ day (table I). In six patients who had been on a combination of phenformin and ethyloestrenol, phenformin was withdrawn because of increased serum creatinine levels. Treatment with ethyloestrenol continued in a dose of $8 \mathrm{mg} /$ day. The patients were followed up for recurrences of thrombosis and for side effects.

Fifteen patients (aged 35-82 years; mean $64 \cdot 7$ years) with a normal fibrinolytic defence mechanism were also studied. Four had had recurrent DVT (two to five episodes each) and the remaining 11 had severe arteriosclerosis. These patients were given ethyloestrenol $8 \mathrm{mg} /$ day and were re-examined after three, six, and 12 months.

Laboratory methods-The spontaneous fibrinolytic activity of resuspended euglobulin precipitate of plasma was measured on unheated fibrin plates ${ }^{7}$ (normal range: $0-70 \mathrm{~mm}^{2}$ ).

Venous occlusion was performed as described. ${ }^{4} \mathrm{~A}$ sphygmomanometer cuff was placed around each upper arm and inflated to a pressure between the systolic and diastolic blood pressure for 20 minutes. Blood samples for determining the fibrinolytic activity of resuspended euglobulin precipitate on fibrin plates were withdrawn before the cuff was applied and again just before it was deflated. The mean of the fibrinolytic activity in the samples withdrawn at the end of occlusion from each arm was taken as a measure of the capacity of releasing the fibrinolytic activators from the vessel wall. In 118 apparently healthy volunteers aged $18-50$, the 5 th percentile was $158 \mathrm{~mm}^{2}\left(95 \%\right.$ confidence interval 88-169 $\left.\mathrm{mm}^{2}\right)$.

Fibrinolytic activity in superficial hand veins-Biopsy specimens excised from a superficial hand vein under local anaesthesia $(0.5 \%$ mepivacaine hydrochloride) were examined by Pandolfi's modification of Todd's fibrinolysis autography technique. ${ }^{89}$ The fibrinolytic activity was expressed in arbitrary units. ${ }^{5}$ The median value found at our laboratory in 70 healthy volunteers was 7.5 arbitrary units (range 6-10).

Fibrinogen concentrations were determined according to the syneresis method of Nilsson and Olow (normal range: $2 \cdot 0-4 \cdot 0 \mathrm{~g} / \mathrm{l}$ ).

Statistical methods-The significance of differences was calculated by Student's $t$ test. The difference between the biopsy findings was, however, analysed by Wilcoxon's sum rank test.

\section{Results}

The spontaneous fibrinolytic activity of circulating blood was significantly increased after three months' treatment $(P<0.001)$ and

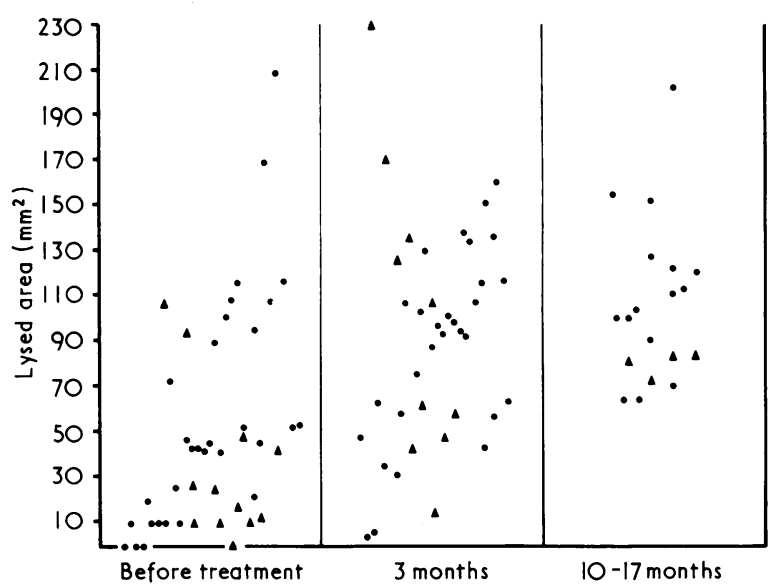

FIG 1-Spontaneous fibrinolytic activity (resuspended euglobulin precipitate of plasma on unheated plates) during treatment with ethyloestrenol $8 \mathrm{mg} /$ day $(\bigcirc)$ and $4 \mathrm{mg} /$ day $(\Delta)$.

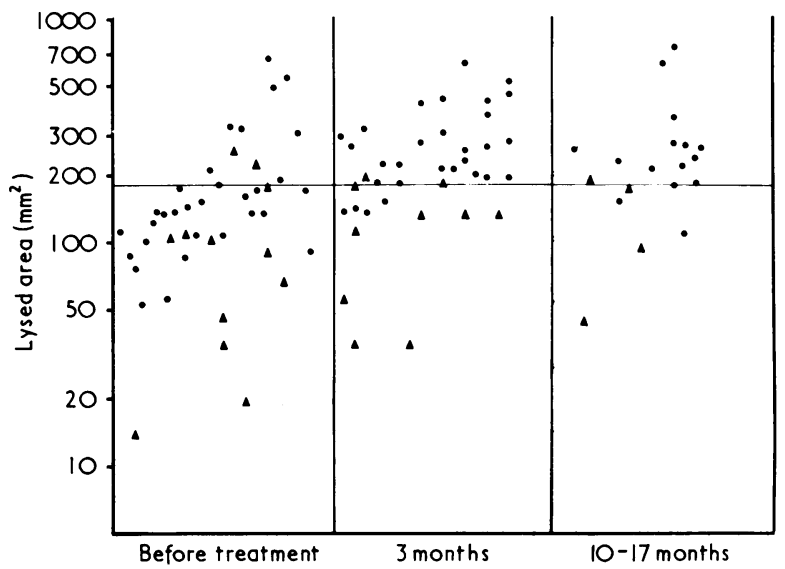

FIG 2-Local fibrinolytic activity during venous occlusion of arms during treatment with ethyloestrenol $8 \mathrm{mg} /$ day $(O)$ and $4 \mathrm{mg} /$ day (A). Horizontal line indicates mean fibrinolytic activity of resuspended euglobulin precipitate on unheated fibrin plates of both arms.

TABLE II-Number of patients with decreased fibrinolytic mechanism before and after treatment with ethyloestrenol $8 \mathrm{mg} /$ day or $4 \mathrm{mg} /$ day

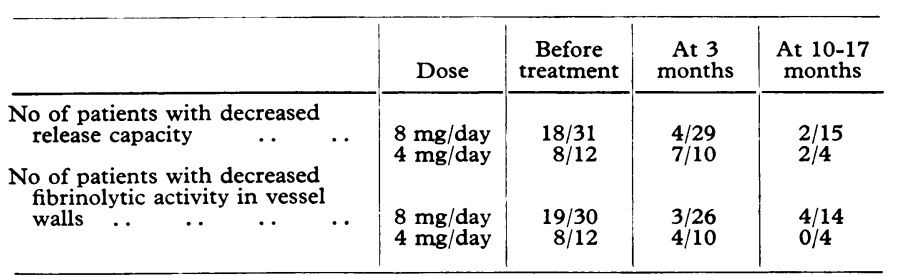


persisted at about the same level throughout treatment on both doses of ethyloestrenol (fig 1). After 10-17 months' treatment the further increase was hardly significant $(P<0.02)$

The release capacity of fibrinolytic activators from the vessel walls, measured as the local fibrinolytic activity obtained after venous occlusion, is given in fig 2 . In 18 out of 31 patients the release capacity was defective before treatment $\left(<158 \mathrm{~mm}^{2}\right)$. After three months' treatment with ethyloestrenol $8 \mathrm{mg} /$ day only four patients still had a decreased release capacity and after 10-17 months' treatment only two of the 15 examined patients had a decreased release capacity (fig 2; table II). After three months' treatment with ethyloestrenol $8 \mathrm{mg} /$ day a significant increase $(P<0.01)$ was obtained. No further increase was seen $(P>0.05)$ during treatment. No significant increase occurred in any of the 15 patients who had a normal release capacity before the treatment (see table III). No significant increase in the fibrinolytic capacity was observed after treatment with $4 \mathrm{mg}$ /day over three to 17 months (table II).

TABLE III-Effect of ethyloestrenol $8 \mathrm{mg} /$ day in patients with normal fibrinolytic systems before treatment

\begin{tabular}{|c|c|c|c|}
\hline & $\begin{array}{l}\text { Before } \\
\text { treatment }\end{array}$ & $\begin{array}{l}\text { After three } \\
\text { months' } \\
\text { treatment }\end{array}$ & $\begin{array}{l}\text { After } 12 \\
\text { months' } \\
\text { treatment }\end{array}$ \\
\hline $\begin{array}{l}\text { Spontaneous fibrinolytic } \\
\text { activity }\left(\mathrm{mm}^{2}\right) \\
\text { Release capacity }\left(\mathrm{mm}^{2}\right)\end{array}$ & $\begin{array}{l}110 \\
312\end{array}$ & $\begin{array}{l}124 \\
359\end{array}$ & $\begin{array}{l}149 \\
309\end{array}$ \\
\hline $\begin{array}{l}\text { Fibrinolytic activity in vessel } \\
\text { walls (arbitrary units) }\end{array}$ & $7 \cdot 0$ & 7.5 & 8.0 \\
\hline
\end{tabular}

The fibrinolytic activity in the vessel walls was decreased $(<6$ arbitrary units) in 19 out of 30 patients before treatment with ethyloestrenol $8 \mathrm{mg} /$ day (fig 3). The mean level of the fibrinolytic activity rose significantly after three months' treatment $(P<0.001)$ and the mean remained at about 7 arbitrary units throughout the observation period. After three months' treatment three patients out of 26 examined still had a decreased vessel wall fibrinolytic activity, and after 10-17 months four out of 14 still showed a decreased level ( $<6$ arbitrary units) (table II)

After treatment with $4 \mathrm{mg} /$ day (fig 3) vessel wall activity did not increase significantly until after 10 to 15 months $(P<0.01)$. Before treatment eight out of 12 patients showed decreased activity in the vessel walls and four out of 10 showed decreased activity after three months' treatment $(P>0.05)$. After 10 to 15 months' treatment none of the four patients examined showed a decreased vessel wall activity (table II).

The patients who had earlier been treated with phenformin and ethyloestrenol ( $8 \mathrm{mg} /$ day) all had a normal fibrinolytic system when phenformin was withdrawn. During the subsequent treatment with only ethyloestrenol ( $8 \mathrm{mg} /$ day) they remained normal.

Spontaneous fibrinolytic activity, release capacity after venous occlusion, and fibrinolytic activity in the vessel walls did not increase significantly in patients with a normal fibrinolytic defence mechanism (table III). No significant changes of the fibrinogen were observed during treatment $(P>0.05)$.

One recurrence occurred within three months of treatment, when the fibrinolytic system was not yet normal. One patient developed suspected resistance. After three months' treatment both fibrinolytic activator activity in the vessel walls and release capacity became normal. At re-examination 11 and 16 months after the start of treatment a decreased vessel wall activity was found.

Side effects-Two of the three women of fertile age developed irregular menstrual bleeding and one of them also claimed to have put on weight. Because of these side effects the treatment was withdrawn, and the menstrual bleeding pattern returned to normal. No other side effects were observed.

\section{Discussion}

Prophylactic treatment with a combination of phenformin and ethyloestrenol normalises the defective fibrinolytic system in patients with a decreased fibrinolytic activity in the vessel walls or a defective release capacity of fibrinolytic activators from the vessel walls, or both. ${ }^{4}$

Severe side effects, such as lactic acidosis, have, however, been reported during treatment with phenformin. ${ }^{1011}$ One of the

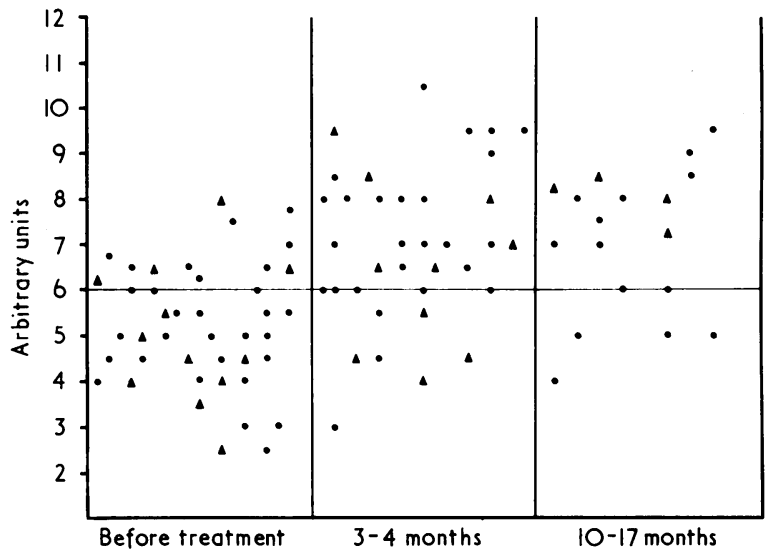

FIG 3-Fibrinolytic activity in vein walls expressed in arbitrary units during treatment with ethyloestrenol $8 \mathrm{mg} /$ day $(O)$ and $4 \mathrm{mg} /$ day $(\Delta)$. Horizontal line represents lower limit of normal.

patients reported by Nilsson et $a^{4}$ also developed this severe complication. Therefore it seemed reasonable to try treatment with the anabolic steroid ethyloestrenol alone. In a dose of $8 \mathrm{mg} /$ day ethyloestrenol significantly increased the spontaneous fibrinolytic activity in the circulation and the release capacity of the fibrinolytic activators from the vessel walls after three months treatment. Nevertheless, no increase occurred in patients who had a normal fibrinolytic system before treatment. The same was observed after combined treatment with ethyloestrenol and phenformin. ${ }^{4}$ Ethyloestrenol thus seems to influence specifically the defective mechanism and not only to induce an overall increase in synthesis of fibrinolytic activator and its release from the vessel wall. Most of the patients with a normal fibrinolytic system had severe arteriosclerosis, however, so the possibility that a damaged vessel wall might have contributed to the lack of response to treatment cannot be excluded. Also the fibrinolytic activity of the vessel walls increased significantly during three months' treatment, but after this initial increase no further increase was observed, which agrees with the findings after the combination therapy. ${ }^{4}$ The lower dose of $4 \mathrm{mg} /$ day did not seem to be sufficient to affect the fibrinolytic system.

In four patients the fibrinolytic activity of the vessel walls did not respond to treatment with $8 \mathrm{mg} / \mathrm{day}$. Only one patient developed a resistance to ethyloestrenol.

No recurrence occurred in the patients with a fibrinolytic system that had returned to normal, which might suggest that the effective treatment that results in a normal fibrinolytic system is effective as thrombosis prophylaxis in patients with a fibrinolytic defect.

This work was supported by grants from the Swedish Medical Research Council (B76-19X-00087-12B) and the Medical Faculty, University of Lund.

\section{References}

1 Isacson, S, and Nilsson, I M, Acta Chirurgica Scandinavica, 1972, 138, 313.

2 Fearnley, G R, Chakrabarti, R, and Hocking, E D, Lancet, 1967, 2, 1008.

${ }^{3}$ Isacson, S, and Nilsson, I M, Scandinavian fournal of Haematology, $1970,7,404$

${ }^{4}$ Nilsson, I M, Hedner, U, and Isacson, S, Acta Medica Scandinavica, $1975,198,107$.

5 Davidson, J F, et al, British fournal of Haematology, 1972, 22, 543.

${ }^{6}$ Davidson, J F, Walker, I D, and McCallum, H I, Progress in Chemical Fibrinolysis and Thrombolysis, 1975, 1, 311

7 Nilsson, I M, and Olow, B, Thrombosis et Diathesis Haemorrhagica, 1962, 8, 297.

${ }^{8}$ Pandolfi, M, Isacson, S, and Nilsson, I M, Acta Medica Scandinavica, 1969, 186, 1 .

9 Pandolfi, M, European Fournal of Clinical and Biological Research, 1972, 17, 254.

10 Wise, P H, et al, British Medical fournal, 1976, 1, 70.

11 MacGregor, G A, Poole-Wilson, P A, and Jones, N F, Lancet, 1972, 1, 69. 